\title{
A proprietary polyphenolic blend offers improved strength recovery and reduced delayed onset muscle soreness post-exercise
}

\author{
Tatania K Emmick*, Kelli A Herrlinger \\ From The Eleventh International Society of Sports Nutrition (ISSN) Conference and Expo \\ Clearwater Beach, FL, USA. 20-21 June 2014
}

\section{Background}

The sports nutrition industry is seeing growth in consumers who are embarking on high intensity daily workout routines to regain and maintain physical fitness. This type of demanding training results in exercise-induced muscle damage setting off a cascade of increased oxidative stress and inflammation which in turn leads to reduced strength and increased delayed onset muscle soreness (DOMS) for days post-training. Consumption of antioxidants and anti-inflammatory molecules is crucial to counteract these negative side effects. A proprietary polyphenolic blend of catechins and theaflavins (PPCT) was formulated and evaluated in a randomized, doubleblind, placebo controlled human trial to determine the effect on exercise performance recovery following eccentric exercise.

\section{Methods}

Male participants (age 18-35 years) volunteered and were randomized to receive either a placebo or PPCT $(2,000 \mathrm{mg})$ daily for 13 weeks. During the $13^{\text {th }}$ week of supplementation, subjects performed a 40 minute downhill treadmill run $\left(65 \%\right.$ of $\left.\mathrm{VO}_{2 \max }\right)$ with 3 sets of isokinetic leg extension measurements taken at baseline (pre-exercise), 24, 48 and 96 hours post-exercise. Delayed onset muscle soreness (DOMS), muscle damage via creatine kinase (CK), oxidative stress via ferric reducing antioxidant power (FRAP), and a stress hormone (cortisol) were also examined at these timepoints. Consent to publish the results was obtained from all participants.

\section{Results}

The treatment group regained strength as measured by peak torque to $98 \%$ and $101 \%$ of pre-exercise levels at 48 and 96 hours post-exercise, respectively. In comparison the placebo group's peak torque levels remained at $92 \%$ and $93 \%$ of pre-exercise levels at the same time points post-exercise. These improvements were significant compared to placebo at both time points $(\mathrm{p}<0.05)$. Additionally, participants in the PPCT group reported decreased whole body and hamstring DOMS compared to placebo at 48 hours ( $p=0.029$ for both). These enhancements in strength and DOMS were also supported by improvements in serum markers of oxidative stress, muscle damage and inflammation. Chronic consumption of PPCT improved serum antioxidant status $(\mathrm{p}=0.039)$ as measured by FRAP. As expected, serum cortisol increased in all groups compared to pre-exercise levels; however by 96 hours, serum cortisol levels had returned to pre-exercise levels in the PPCT group while the placebo remained 20\% above pre-exercise levels $(\mathrm{p}<0.05)$. Creatine kinase (CK) increased in both groups peaking at 24 hours post-exercise. CK levels returned to pre-exercise values at 96 hours in the PPCT groups while levels in the placebo group remained significantly elevated $50 \%$ over pre-exercise levels $(\mathrm{p}<0.05)$ at the same time point. These reductions in cortisol and CPK levels occur simultaneous to the recovery in pre-exercise strength observed at 96 hours.

\section{Conclusions}

Daily supplementation with PPCT was shown to reduce DOMS and promote recovery of muscle strength by reducing the oxidative stress and markers of muscle damage that occurs post-exercise.

\footnotetext{
* Correspondence: Tatania.Emmick@kemin.com
} Kemin Foods, L.C., Des Moines, lowa, USA 


\section{Acknowledgement}

Tatania Emmick and Kelli Herrlinger are employed by Kemin Foods, L.C., the sponsor of the aforementioned study.

Published: 1 December 2014

doi:10.1186/1550-2783-11-S1-P10

Cite this article as: Emmick and Herrlinger: A proprietary polyphenolic blend offers improved strength recovery and reduced delayed onset muscle soreness post-exercise. Journal of the International Society of Sports Nutrition 2014 11(Suppl 1):P10.

Submit your next manuscript to BioMed Central and take full advantage of:

- Convenient online submission

- Thorough peer review

- No space constraints or color figure charges

- Immediate publication on acceptance

- Inclusion in PubMed, CAS, Scopus and Google Scholar

- Research which is freely available for redistribution

Submit your manuscript at www.biomedcentral.com/submit
() Biomed Central 